OPEN ACCESS

Edited by:

Michael W. Berns,

University of California, Irvine,

United States

Reviewed by:

Chengbiao $\mathrm{Wu}$

California College San Diego,

United States

Nicole M. Wakida,

University of California, Irvine,

United States

*Correspondence:

Vincent R. Daria

vincent.daria@anu.edu.au

Specialty section:

This article was submitted to Optics and Photonics,

a section of the journal

Frontiers in Physics

Received: 31 August 2020

Accepted: 27 October 2020

Published: 26 November 2020

Citation:

Castañares ML, Bachor H-A and Daria VR (2020) Analyzing Branch-specific Dendritic Spikes Using an Ultrafast

Laser Scalpel.

Front. Phys. 8:600971.

doi: 10.3389/fphy.2020.600971

\section{Analyzing Branch-specific Dendritic Spikes Using an Ultrafast Laser Scalpel}

\author{
Michael L. Castañares ${ }^{1}$, Hans-A. Bachor ${ }^{2}$ and Vincent R. Daria ${ }^{1,2 *}$ \\ ${ }^{1}$ John Curtin School of Medical Research, Australian National University, Canberra, ACT, Australia, ${ }^{2}$ Research School of Physics, \\ Australian National University, Canberra, ACT, Australia
}

Dendritic spikes facilitate neuronal computation and they have been reported to occur in various regions of the dendritic tree of cortical neurons. Spikes that occur only on a select few branches are particularly difficult to analyze especially in complex and intertwined dendritic arborizations where highly localized application of pharmacological blocking agents is not feasible. Here, we present a technique based on highly targeted dendrotomy to tease out and study dendritic spikes that occur in oblique branches of cortical layer five pyramidal neurons. We first analyze the effect of cutting dendrites in silico and then confirmed in vitro using an ultrafast laser scalpel. A dendritic spike evoked in an oblique branch manifests at the soma as an increase in the afterdepolarization (ADP). The spikes are branch-specific since not all but only a few oblique dendrites are observed to evoke spikes. Both our model and experiments show that cutting certain oblique branches, where dendritic spikes are evoked, curtailed the increase in the ADP. On the other hand, cutting neighboring oblique branches that do not evoke spikes maintained the ADP. Our results show that highly targeted dendrotomy can facilitate causal analysis of how branchspecific dendritic spikes influence neuronal output.

Keywords: laser scissors, dendritic spike, pyramidal neuron, dendrites, two-photon imaging, compartmental modeling

\section{INTRODUCTION}

Dendrites perform computation with the aid of dendritic spikes (D-spikes) [1-3]. A D-spike is characterized by a nonlinear intensification of the membrane potential caused by regenerative activation of voltage-gated ion-channels. The broad temporal profiles of D-spikes $(>20 \mathrm{~ms})$ enable neurons to boost their firing probability with coincident synaptic inputs occurring within a broad time window $[4,5]$. Hence, coincidence detection with the aid of D-spikes presents an important computing mechanism in pyramidal neurons $[6,7]$. In vitro studies have shown that D-spikes occur in cortical pyramidal neurons [8-10] as well as hippocampal neurons [11-14] and their role in single neuron computation impacts information processing in neuronal circuits collectively.

D-spikes have been observed in several locations in the dendritic tree of cortical pyramidal neurons. In layer five pyramidal neurons (L5PNs), D-spikes mediated by voltage-gated calcium channels (VGCCs) have been reported to occur at the nexus of the apical tuft dendrites, which causes a global depolarization of the main apical trunk and apical branches [10, 15, 16]. Moreover, N-Methyl-D-Aspartate (NMDA) spikes have been reported to occur at the apical tuft [17] and basal dendrites $[18,19]$ of pyramidal neurons. 
We have recently reported that $\mathrm{D}$-spikes also occur in apical oblique dendrites of L5PNs [20, 21]. D-spikes in apical oblique branches are triggered by a low-frequency $(f \geq 60 \mathrm{~Hz})$ train of action potentials (APs), which we initially investigated using a multi-compartment model of a L5PN and confirmed experimentally via functional calcium imaging [21]. As APs back-propagate to the dendritic tree, they activate VGCCs at the dendrite to generate a spike. We have numerically characterized the $\mathrm{D}$-spike in oblique branches as a fast sodium spike followed by a broad depolarization due to regenerative activation high-voltage activated calcium channels [20].

D-spikes can be investigated by pharmacologically blocking certain channels along specific regions of the dendritic tree [17, $22,23]$. However, targeted and highly-localized drug delivery is challenging to perform especially on thin and complex dendritic morphologies such as the apical oblique branches of L5PNs. Neighboring dendrites could be unnecessarily applied with drugs and therefore not viable for analyzing D-spikes that occur only in a few among many intertwined dendrites.

An alternative way to investigate D-spikes is by dendrotomy. Bekkers and Häusser [24] used a pair of micropipettes to pinch the main apical trunk and to study its active and passive properties. Although their work did not focus on D-spikes evoked in particular dendritic domains, their findings show that dendrotomy of the apical trunk of L5PNs reduced the overall excitability and the afterdepolarization (ADP) at the soma. While the ADP comes from multiple sources across the dendritic tree [25], it has been primarily associated with the recruitment of D-spikes in specific regions such as the nexus of the apical tuft $[15,16]$ and oblique branches of L5PNs [21]. However, unlike the wide reach of a D-spike at the nexus that extends throughout the tuft, investigating spikes in select oblique branches requires identifying the specificity of spike generation. Which among the oblique dendrites are most likely to produce a spike? To answer this question, we need a tool that allows us to tease out the influence of certain branches to the overall neuronal function.

Here, we aim to use dendrotomy to investigate branch-specific D-spikes in apical oblique dendrites of L5PNs. To aid our analysis, we numerically simulated the process and implemented dendrotomy in vitro using L5PNs in acute brain slices. While the technique used by Bekkers and Häusser [24] has been effective for dendrotomy of thick dendrites (e.g., apical trunk), using micropipettes to pinch thin obliques without affecting its neighboring dendrites can be very challenging. Hence, we adopted a highly-targeted optical ablation approach using a tightly-focused ultrafast laser scalpel [26]. We evoked D-spikes in apical oblique dendrites [21] and observed the changes in the ADP profile following dendrotomy. Since dendrotomy via an ultrafast laser scalpel is an irreversible process, it would take a large number of experiments to draw neuro-physiological conclusions as to the extent of specificity of spike generation among oblique branches. Nonetheless, our results provide the groundwork for using a laser scalpel to tease out the computing mechanisms of certain branches among complex dendritic arborizations in cortical pyramidal neurons.

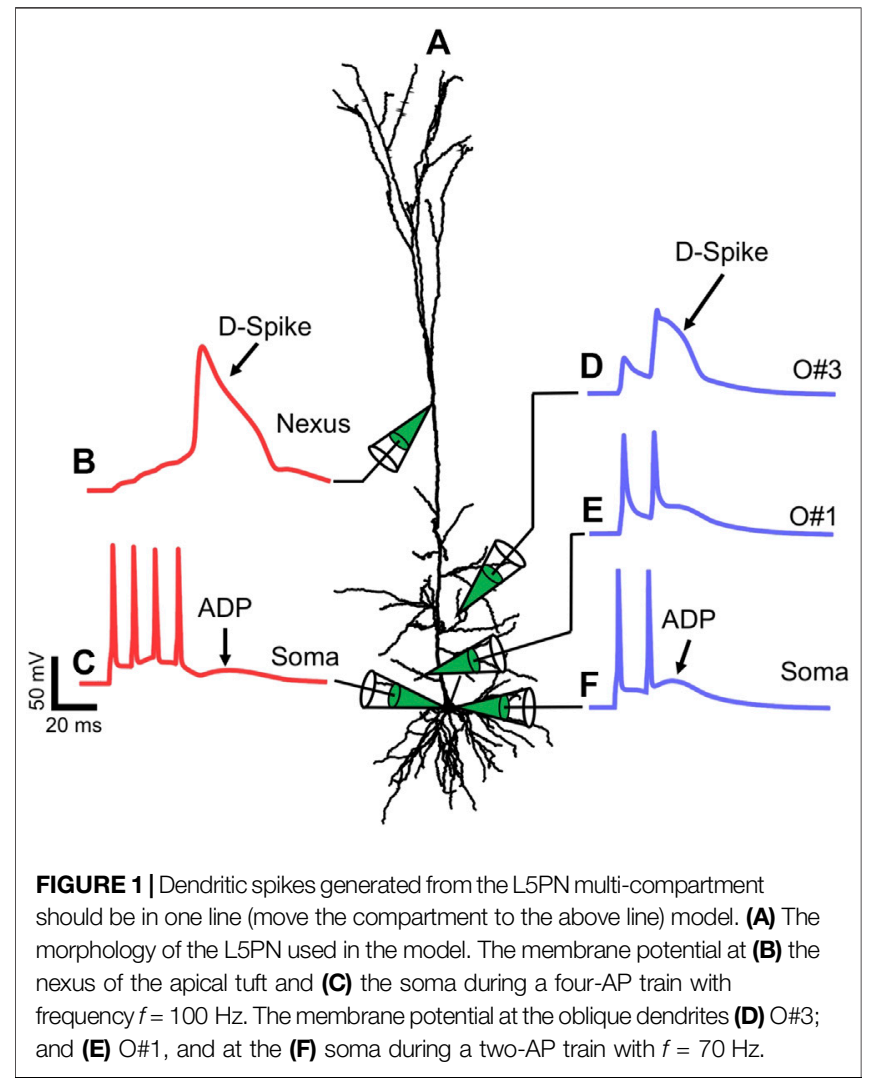

\section{MULTI-COMPARTMENTAL MODEL}

We implemented our numerical experiments via NEURON and Python [27] using a multi-compartmental model of an L5PN shown in Figure 1A [28]. The procedure for numerical experiments is described in the Supplementary Methods 1. We slightly modified the L5PN model to shape the neuron's response closer to our empirical data [21]. Our experiments show that oblique branches of L5PNs are less excitable compared to the model. Hence, we incorporated potassium $\left(\mathrm{K}^{+}\right)$ion-channels (fast-activating $\left(K_{\mathrm{T}}\right)$ and persistent-activating $\left(K_{\mathrm{PST}}\right) \mathrm{K}^{+}$ channels) to regulate dendritic excitability and control the extent of backpropagating APs, as previously implemented in models [29-31], and experiments involving L5PNs [31,32] and hippocampal neurons [13, 22]. Detailed description of the modified model is described in Supplementary Methods 2.

To generate D-spikes, we injected brief current pulses (Amplitude 3-4 nA, Pulse-width $2 \mathrm{~ms}$ ) at the soma to elicit a train of APs. The frequency of the train of two or four APs ranges from $20 \leq f \leq 120 \mathrm{~Hz}$. When the APs back-propagate to the dendrites, they can initiate D-spikes at certain areas of the dendritic tree. For example, a train of four action potentials (four-AP train) with $f=100 \mathrm{~Hz}$ can initiate a D-spike at the nexus of the apical tuft dendrites [15]. Figure 1B shows the trace of the membrane potential, $V_{\mathrm{m}}$, at the nexus of the apical tuft during a four-AP train while Figure 1C shows the $V_{\mathrm{m}}$ at the soma. On the other hand, a two-AP train elicits a D-spike at certain oblique branches as shown in Figure 1D and 

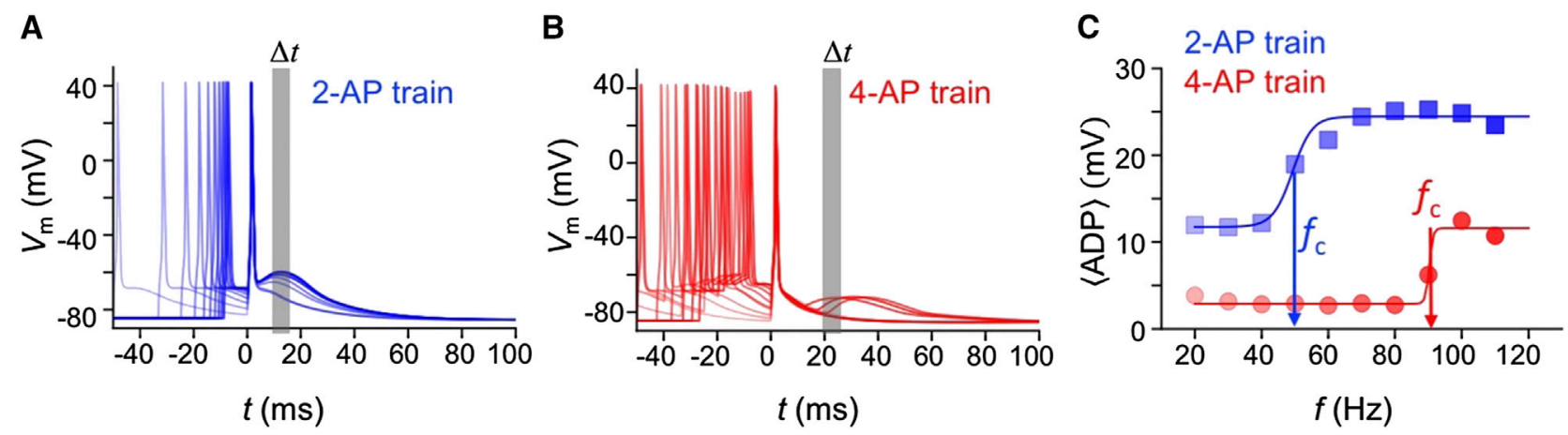

FIGURE 2 | After depolarization associated with D-spikes. The somatic membrane potential aligned to the last AP, following (A) two-AP, and (B) four-AP trains with frequencies from $20 \leq f \leq 120 \mathrm{~Hz}$. (C) The frequency response of the average ADP, $\langle A D P\rangle$, taken over $\Delta$ t during two-AP (blue squares) and four-AP trains (red circles).

corresponding somatic $V_{\mathrm{m}}$ recording shown in Figure 1F. When a D-spike is generated, the broad temporal depolarization of the spike manifests at the soma as an ADP that follows after the last AP of the train. Somatic $V_{\mathrm{m}}$ recordings in Figures 1C,F show traces with a slight bump following the last AP. While there are multiple sources of the ADP, a larger ADP is observed when the AP train evokes a D-spike in one or multiple dendrites. Note that not all oblique branches evoke a D-spike during a two-AP train. Other dendrites, such as the proximal oblique $\mathrm{O} \# 1$, do not evoke a D-spike as observed by the absence of a temporally broad depolarization (Figure 1E). In this particular L5PN model, branch-specific D-spikes are evoked only at two oblique dendrites: O\#3 (Figure 1D) and O\#5 (data not shown) [21].

The minimum frequency of the AP train required to generate the $\mathrm{D}$-spike is referred to as the critical frequency, $f_{\mathrm{c}}$ [15]. The $f_{\mathrm{c}}$ is derived by plotting the neuron's response (dendritic or somatic ADP recording) as a function of frequency. The frequency response is fitted with a Sigmoid function and $f_{\mathrm{c}}$ is the inflection point where the step increase in the neuron's response occurs. Electrophysiological recording at the dendrite where the spike occurs provides for an accurate characterization of D-spikes but could be challenging especially when the dendrite is thinner than the tip of the electrode. Optical recording via calcium or voltage indicators can also be used to record dendritic activity [33]. However, both optical and electrophysiological approaches to record dendritic activity become ineffective after dendrites are severed from the neuron. Hence, for this work, we use electrophysiological recording at the soma to characterize D-spikes based on the changes in the ADP profile before and after dendrotomy. Note, however, that the ADP is a net depolarization from multiple sources in the neuron's dendritic tree [25] and is therefore ambiguous to use it solely to identify branches where D-spikes occur. Nonetheless, we have prior work that shows calcium responses recorded from oblique branches showing D-spikes from these dendrites are correlated with ADP profiles at the soma [21].

We measured the ADP from the somatic recordings to extract the critical frequencies of the dendritic spikes. Figures $\mathbf{2 A , B}$ are voltage traces taken from the soma of the L5PN model during two-AP and four-AP trains, respectively, with the traces aligned to the peak of the last $\mathrm{AP}(t=0)$. The average ADP is calculated as $\langle A D P\rangle=(\Delta t)^{-1} \int_{t_{o}}^{t_{0}+\Delta t} V_{m} d t$, where $\Delta t=5 \mathrm{~ms}$ is the timewindow used for averaging the ADP from $\mathrm{t}_{\mathrm{o}}=10 \mathrm{~ms}$ (for two$A P$ train) and $\mathrm{t}_{\mathrm{o}}=20 \mathrm{~ms}$ (for four-AP train). Figure $2 \mathrm{C}$ shows frequency responses of the $\langle A D P\rangle$. The plots are fitted with a Sigmoid function $\left(S(f)=A /\left(1+\exp \left[-\beta\left(f-f_{c}\right)\right]\right)\right.$, to obtain critical frequencies, $f_{c}=50 \mathrm{~Hz}$ and $f_{c}=90 \mathrm{~Hz}$, following twoAP and four-AP train stimulation, respectively. The fitting parameters, $A(\mathrm{mV})$ and $\beta\left(\mathrm{Hz}^{-1}\right)$, refer to the amplitude and slope of the Sigmoid function, which can be used to evaluate the non linearity of the frequency response.

\section{OBLIQUE BRANCH SPIKE IN L5PNS IN VITRO}

We prepared $300-\mu \mathrm{m}$-thick brain slices from Wistar male rats (P26-P34 days old). Prior to slicing, the rats were sedated by inhalation of $2-4 \%$ isoflurane with oxygen (with flow of $3 \mathrm{~L} / \mathrm{min}$ ) and decapitated according to the protocol approved by the Animal Experimentation Ethics Committee of the Australian National University (Ethics Protocol ID: A2018/35). Somatosensory cortical brain slices were prepared using a vibratome and perfused with oxygenated $\left(\begin{array}{lllll}95 \% & \mathrm{O}_{2} / 5 \% & \mathrm{CO}_{2}\end{array}\right)$ extracellular solution containing (in $\mathrm{mM}$ ): $125 \mathrm{NaCl}, 25 \mathrm{NaHCO}_{3}, 25$ glucose, $3 \mathrm{KCl}, 1.25 \mathrm{NaH}_{2} \mathrm{PO}_{4}, 2 \mathrm{CaCl}_{2}, 1 \mathrm{MgCl}_{2}, \mathrm{pH}$ 7.4. Whole-cell patchclamp recordings were made with 4-6 $\mathrm{M} \Omega$ pipettes filled with an intracellular solution containing (in $\mathrm{mM}$ ): $115 \mathrm{~K}$-gluconate, $20 \mathrm{KCl}$, 10 HEPES, 10 phosphocreatine, 4.0 ATP-Mg, 0.3 GTP, and 0.13 Alexa Fluor 488 dye (Sigma Aldrich) [33].

For patch-clamp recordings and rendering of the neuron's dendritic tree, the tissue sample was transferred to our custombuilt two-photon (2P) microscope shown in Figure 3A [26, 34]. The microscope uses a near-infrared femtosecond laser (Chameleon, Coherent Scientific) and galvanometer scanning mirrors to render a three-dimensional (3D) image of the patched neuron. The microscope is built from a standard differential interference contrast microscope (Olympus BX50) and modified to incorporate a two-photon and holographic projection function [34]. While the microscope has other modalities (e.g., holographic projection), this work only used the mechanical two- 

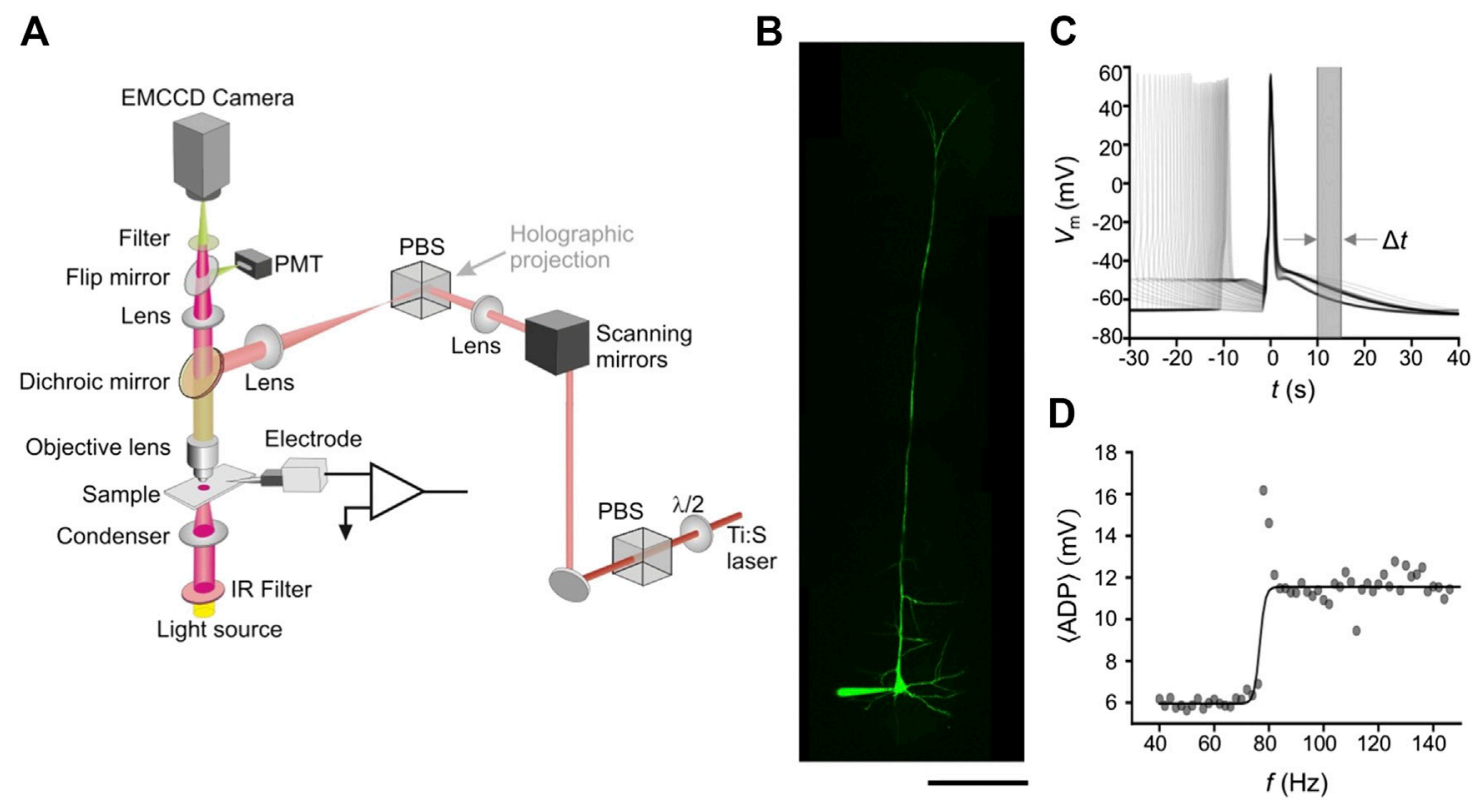

FIGURE 3 | Experimental setup and electrophysiological recordings: (A) Schematic of our custom-built two-photon microscope used for laser dendrotomy. Different modalities are merged using a polarizing beam splitter (PBS), while the emitted fluorescence is acquired via a photomultiplier tube (PMT). (B) A flattened image stack of an L5PN intracellularly loaded with Alexa Fluor 488 dye. (C) The membrane potential recorded from the soma following a two-AP train with frequencies from 40 to $150 \mathrm{~Hz}$ aligned to the second AP (D) The frequency response of the ADP from the recordings in (C). Scale bar in (B) is $50 \mu \mathrm{m}$.

dimensional (2D) beam scanners for imaging and positioning the laser focus onto a dendrite. Figure 3B shows a representative image of a L5PN loaded with Alexa Fluor 488.

After mapping the 3D morphology of the entire neuron, we used the same protocol as our model to determine the critical frequencies from the changes in the ADP profile. We injected two pulse trains (Amplitude of 3-4 nA, Pulse-width of $2 \mathrm{~ms}$ ) to produce a two-AP train with frequencies ranging from $4<f<150 \mathrm{~Hz}$ and the somatic recording is shown in Figure 3C. We took the $\langle A D P\rangle$ over $\Delta \mathrm{t}$ and the frequency response of the $\langle A D P\rangle$ shows a non linear step increase at the critical frequency (Figure 3D). We fitted a Sigmoid function on the frequency response of the $\langle A D P\rangle$ and identified L5PNs that exhibited D-spikes.

\section{DENDROTOMY OF OBLIQUE BRANCHES}

\section{Model}

To perform dendrotomy in silico, dendritic segments were disconnected from the model. We analyzed the temporal profiles of the membrane potentials at the soma during two-AP trains and investigated the changes in the $\mathrm{ADP}$ before and after cutting certain oblique branches. If the increase in the ADP is associated with the generation of a D-spike in a particular oblique branch, we hypothesize that removing that branch alters the ADP profile. On the other hand, removal of an oblique branch that do not support a D-spike, maintains the ADP profile as well as the frequency response of the $\langle A D P\rangle$.

Our numerical experiments show that dendrotomy of certain oblique branches variably affected the ADP and confirming our hypothesis. Cutting a branch that evokes a D-spike (e.g., O\#3, Figure 4A) altered the ADP profile. Figure 4B shows traces of the somatic $V_{\mathrm{m}}$ at various frequencies of the two-AP train (aligned with the second AP) for an intact dendritic tree. The frequency response of the $\langle A D P\rangle$ is fitted with a sigmoid function and shows a non linear step increase occurring at $f_{\mathrm{c}}=50 \mathrm{~Hz}$ (Figure 4C). The fitting parameters of the sigmoid function resulted in an amplitude, $A=13 \pm 1 \mathrm{mV}$ and slope, $\beta=$ $0.22 \pm 0.09 \mathrm{~Hz}^{-1}$. Cutting of $\mathrm{O} \# 3$ altered the ADP profile (Figure 4D) and consequently the frequency response of the $\langle A D P\rangle$ (Figure 4E). The increase in the $\langle A D P\rangle$ at frequencies less than $40 \mathrm{~Hz}$ is potentially due its extended branch morphology, which sets the branch to act as strong sink during back-propagation of an AP. The removal of $\mathrm{O} \# 3$ potentially increased the efficacy of AP invasion to other apical dendrites resulting to a larger somatic depolarization. At frequencies greater than $40 \mathrm{~Hz}$, the $\langle A D P\rangle$ follows a trend that can be fitted with a sigmoid function with $A=8 \pm 1 \mathrm{mV}$ and $\beta=$ $0.10 \pm 0.03 \mathrm{~Hz}^{-1}$. The changes in the fitting parameters before (Pre) and after (Post) dendrotomy of $\mathrm{O} \# 3$ are quantified in Figure 4F, which shows a significant decrease in $A$ and $\beta$. The error bars represent the uncertainty of the fit.

On the other hand, cutting branch $\mathrm{O \# 1}$ (see Figure 4A) did not change the ADP profile (compare Figures 4G,B) as well as the frequency response of the $\langle A D P\rangle$ (compare Figures $\mathbf{4 H}, \mathbf{C}$ ). Note that $\mathrm{O} \# 1$ did not display a $\mathrm{D}$-spike even at higher frequencies of the two-AP train (Figure 1E). Likewise, the changes in the fitting parameters before and after dendrotomy of $\mathrm{O} \# 1$ are quantified in Figure 4I, which shows a slight increase in the amplitude $(A=14 \pm$ $1 \mathrm{mV})$ while the slope is slightly reduced $\left(\beta=0.16 \pm 0.04 \mathrm{~Hz}^{-1}\right)$. 


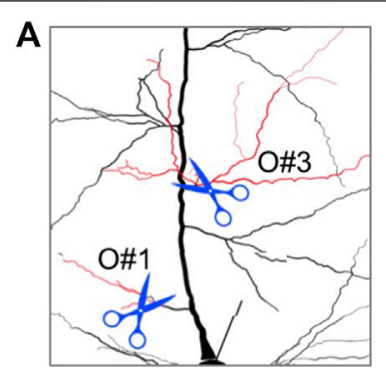

D

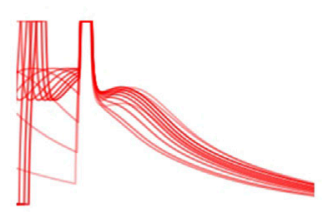

$\mathbf{G}$

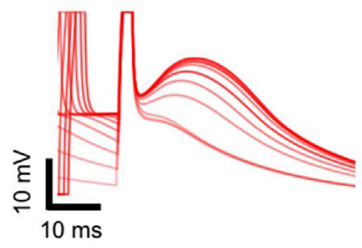

B

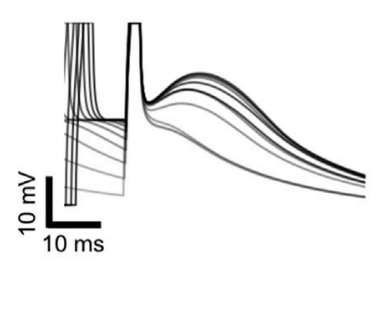

E

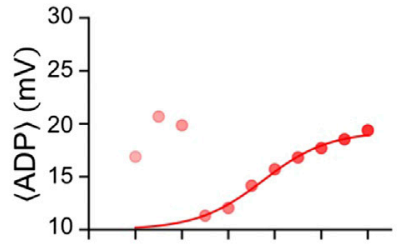

H

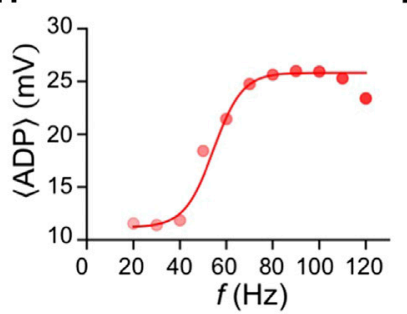

C

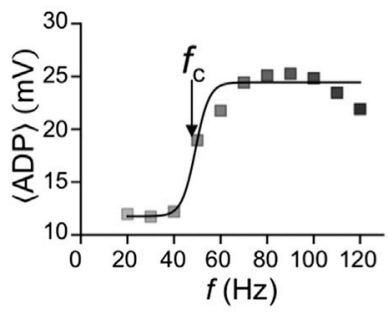

$\mathbf{F}$

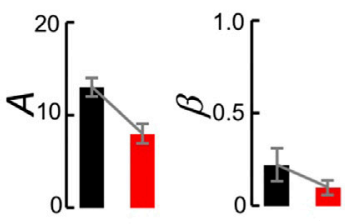

I

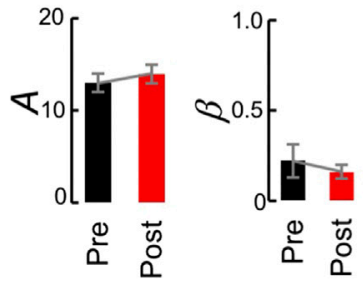

FIGURE 4 | Numerical dendrotomy of oblique branches: (A) A magnified view of the L5PN model following dendrotomy of oblique branches O\#3 or O\#1 (red). (B) Somatic traces during a two-AP train, and (C) corresponding frequency response of the ADP before dendrotomy. (D) Somatic traces after cutting O\#3 and (E) corresponding frequency response of the $\langle A D P\rangle$. (F) Quantification of the changes in fitting parameters, amplitude $(A)$ and slope $(\beta)$, of the Sigmoid function before (Pre) and after (Post) dendrotomy. Cutting oblique branch O\#1 and corresponding (G) somatic traces during a two-AP train, and (H) the frequency response of the $\langle A D P\rangle$ (I) Changes in $A$ and $\beta$ after cutting O\#1. The error bars show uncertainty of the Sigmoid fit.

Differences in neuronal responses when cutting $\mathrm{O} \# 1$ and $\mathrm{O} \# 3$ confirm branch-specific properties of certain oblique dendrites.

\section{Experiment}

We performed targeted dendrotomy using our-custom built $2 \mathrm{P}$ microscope (Figure 3A) by positioning the laser focus onto specific dendrites via the $2 \mathrm{D}$ beam scanners. The transverse resolution of the incision was within the diffraction limit (diameter $\sim 1 \mu \mathrm{m}$ ) matching that of typical diameters of apical oblique branches (Figure 5A). Note that a slight offset of the focus with respect to the dendrite reduced the efficacy of the cut. The average power of the laser used for dendrotomy is $P=80 \mathrm{~mW}$ with an estimated irradiance of $129 \mathrm{~mW} / \mu \mathrm{m}^{2}$ (for 1.0NA Objective), $0.5-3.0 \mathrm{~s}$ exposure time and $\lambda=890 \mathrm{~nm}$. The average power was measured using an optical power meter (Thorlabs) with a photodiode head (S132C, Thorlabs) positioned at the front aperture of the objective lens. We confirmed successful dendrotomy by imaging the targeted dendrite (Figure 5B).

The L5PNs were held in voltage clamp at their typical resting membrane potential of $-65 \mathrm{mV}$. After dendrotomy, the L5PNs depolarized and fired APs, as indicated by a large negative current in the voltage-clamp recording (Figure 5C). The L5PNs were left undisturbed for $10 \mathrm{~min}$ until the holding current, $I_{\mathrm{h}}$, settled to a value that maintained a resting membrane potential of $-65 \mathrm{mV}$ (Figure 5D). The new holding current suggests that the membrane at the cut site has not fully sealed and partial resealing of the cut site caused the neuron to be slightly depolarized after dendrotomy. Despite the change in the holding current, the neurons were still able to fire action potentials when current pulses were injected onto the soma.

We analyzed the ADP of L5PNs before and after dendrotomy. Figure 6 shows somatic recordings before (column A) and after (column B) dendrotomy as well as the frequency response of the $\langle A D P\rangle$ (column C) from four neurons (rows 1-4). The L5PNs exhibited critical frequencies, $f_{\mathrm{c}}=76-100 \mathrm{~Hz}$ during two-AP trains. Dendrotomy of certain oblique branches altered the frequency response of the $\langle A D P\rangle$, which confirmed our hypothesis. Across all neurons, the $\langle A D P\rangle$ decreased by $2-5 \mathrm{mV}$, indicative of a decrease in dendritic excitability (compare columns $\mathrm{A}$ and $\mathrm{B}$ in Figure 6). Column $C$ shows two neurons (\#1 and \#2) with altered frequency response of the $\langle A D P\rangle$. We quantified the changes in the frequency response by comparing the fitting parameters of the Sigmoid function before (Pre) and after (Post) dendrotomy (column D). Prior to cutting, the non linear step increase in the $\langle A D P\rangle$ at their respective critical frequencies yielded amplitudes, $A=5.8 \pm 0.3,4.3 \pm 0.15,2.8 \pm 0.2$ and $7.3 \pm 0.4 \mathrm{mV}$ and slope, $\beta=3 \pm$ $1,0.15 \pm 0.02,0.3 \pm 0.1$ and $0.15 \pm 0.03 \mathrm{~Hz}^{-1}$ from neurons (rows) \#1 
A

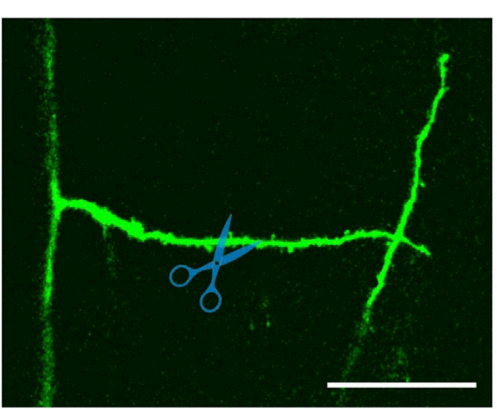

C

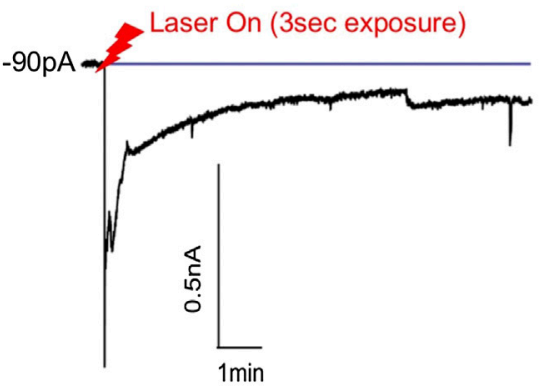

B

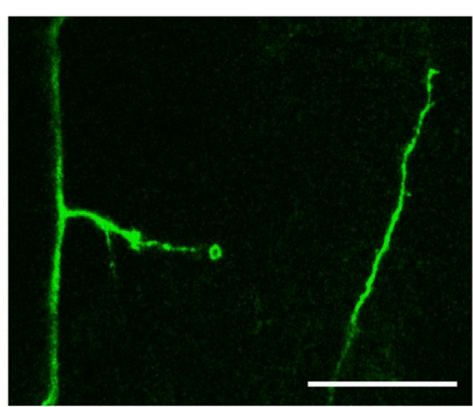

D

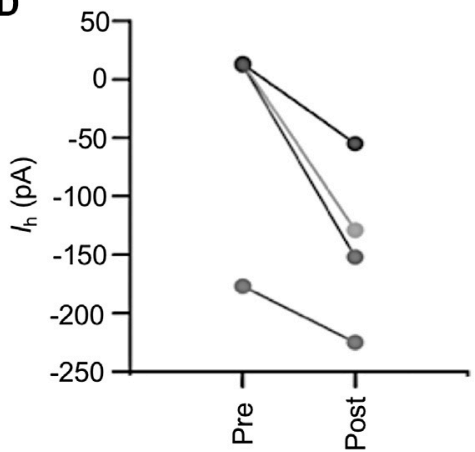

FIGURE 5 | Laser dendrotomy of apical oblique dendrites. Fluorescence image of an oblique branch (A) before dendrotomy, and (B) after dendrotomy, (C) the current recording at the soma. After dendrotomy, the current increased sharply indicating AP firing of the neuron. The current then decayed to a new value after 10 min as membrane at the cut site partially sealed, (D) the holding current before (Pre) and after (Post) dendrotomy required to maintain the membrane potential at -65 mV. Scale bars in $(\mathbf{A})$ and $(\mathbf{B})$ are $10 \mu \mathrm{m}$.

to \#4, respectively. However, cutting an oblique dendrite of two L5PNs reduced the amplitude $A=0.53 \pm 0.08 \mathrm{mV}$ and $1.7 \pm 0.2 \mathrm{mV}$ for neurons $\# 1$ and $\# 2$, respectively. The change in the slope for neuron $\# 1\left(\beta=0.16 \pm 0.09 \mathrm{~Hz}^{-1}\right)$ is significant but not much for neuron $\# 2\left(\beta=0.1 \pm 0.04 \mathrm{~Hz}^{-1}\right)$. Nonetheless, these two neurons resembled to a flattened frequency response after dendrotomy. Such response is representative of cutting $\mathrm{O} \# 3$ in the model (Figure 4).

On the other hand, two L5PNs showed minimal changes in the ADP profile (neurons \#3 and \#4 Figure 6) after dendrotomy. From column D (Post), cutting the proximal oblique dendrites resulted in a slight increase in the amplitude, $A=3.4 \pm 0.2 \mathrm{mV}$ and $11.6 \pm 0.4 \mathrm{mV}$ for neurons \#3 and \#4, respectively. No significant changes in their slopes were observed with $\beta=0.21 \pm 0.05$ (neuron \#3) and $0.14 \pm$ $0.02 \mathrm{~Hz}^{-1}$ (neuron \#4). Such response is representative of cutting the proximal O\#1 branch in the model (Figure 4).

These experimental results agree with our model and suggest that removal of a single dendrite, which potentially evoked a D-spike, affected the overall excitability of L5PNs and altered the frequency response of the $\langle A D P\rangle$. On the other hand, cutting a dendrite that does not support a D-spike, maintained the ADP profile and frequency response of the $\langle A D P\rangle$.

\section{DISCUSSION}

We investigated the influence of branch-specific D-spikes to neuronal function by pruning certain oblique branches of cortical
L5PNs. Using a multi-compartment model of a L5PN, we removed segments of the oblique branches and observed the changes in neuronal output via the frequency response of the $\langle A D P\rangle$. We then confirmed the results of the model by using an ultrafast laser scalpel for cutting apical oblique branches in vitro. In the model, cutting branches that exhibited D-spikes resulted in the reduction of the $\langle A D P\rangle$ at frequencies above $f c$. Experimentally, we observed neurons that exhibited flattening of the $\langle A D P\rangle$ frequency response after laser dendrotomy of an oblique branch. Moreover, we also observed neurons that showed no change in the ADP profile after laser dendrotomy and they represent conditions in our model where we cut a branch that did not exhibit a D-spike.

\section{Dendrotomy and Limitations}

Dendritic branching strongly influences AP-firing [35], AP backpropagation [36], and the coupling of somatic-AP spike and $\mathrm{Ca}^{2+}-\mathrm{AP}$ spike zone at the nexus [5]. The ability to manipulate the neuron's dendritic morphology with the use of micropipettes [24, 37] or laser $[26,38,39]$, is important to understand the relation between structure and function. Laser dendrotomy offers a flexible approach since the focus of the laser can be positioned to target different branches of the neuron. We were able to adapt the set of laser parameters reported previously by Go et al. [26] to prune thin oblique branches while minimizing optically-induced damage on the L5PNs.

The main drawback with our approach is the fact that dendrotomy is an irreversible process. As such, using the technique will require data from several neurons in order to 

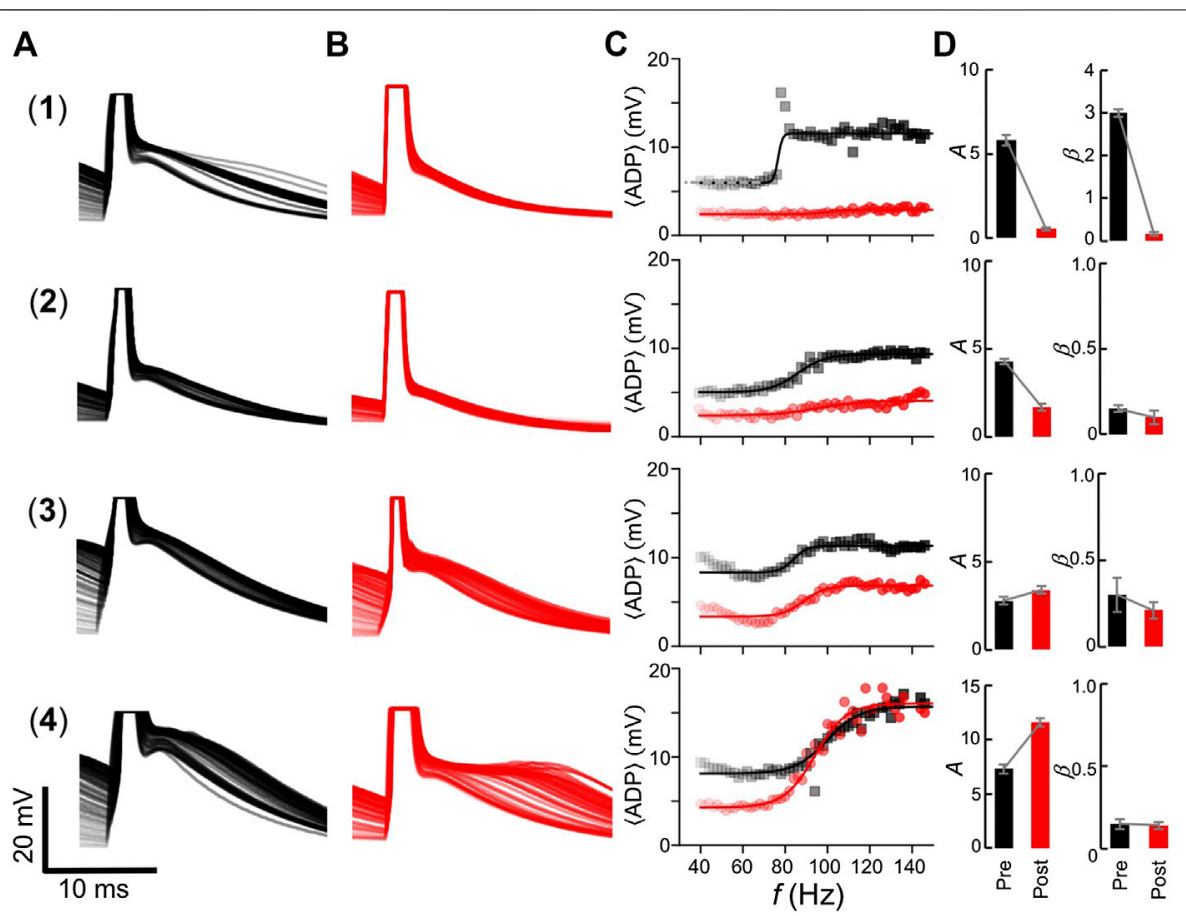

FIGURE 6 |ADP profile and frequency responses before and after dendrotomy. Recordings from four L5PNs (rows 1-4) showing: the somatic membrane potential traces before (column A) and after (column B) dendrotomy aligned to the last AP; (column $\mathbf{C}$ ) the corresponding frequency response of the ADP; and (column $\mathbf{D}$ ) the changes in the fitting parameters of the Sigmoid function, amplitude $(A)$ and slope $(\beta)$, before (pre) and after (post) dendrotomy. The error bars show uncertainty of the Sigmoid fit.

draw statistically significant conclusions on the function of the dendrites as well as their impact on the physiology of neurons as a whole. Moreover, the removal of too much dendritic segments may affect the overall excitability of the neuron. Oblique branches act as a sink for currents propagating along the main apical trunk. The number of oblique branches and their distribution along the trunk determine how effective APs back-propagate up to the nexus of the apical tuft dendrites [5].

\section{Branch-Specific Spikes}

Branch-specific D-spikes in apical oblique branches are recent addition to the well-documented spikes in other regions of the L5PN's dendritic tree [15, 17, 40,41]. The properties of branch-specific D-spikes are not yet well studied. Here, we investigated how D-spikes impact neuronal output and found that cutting certain oblique branches was sufficient to alter the neurons output profile. In summary, our results suggest that: (1) a single oblique branch among many neighboring branches in L5PNs can accommodate a D-spike; (2) a D-spike evoked at the branch manifests at the soma as an increase in the ADP; and (3) cutting an oblique branch that evoked a D-spike changes the ADP profile at the soma.

However, it remains an interesting question as to the role of branch-specific D-spikes in dendritic computation. One potential role is that $\mathrm{D}$-spikes could be a mechanism to raise intracellular calcium levels and promote branchspecific plasticity [42]. Moreover, apart from their role on the dendritic computation, the resultant $\mathrm{ADP}$ at the soma could increase the probability of firing APs (if the ADP reaches firing threshold) and thus influence the excitability of the entire neuron.

\section{Ultrafast Laser Scalpel}

Cutting cellular features with minimal damage to neighboring structures and extracellular medium can be achieved using a tightly focused femtosecond-pulse laser. Cutting is confined within a small interaction volume at the focus where cascades of non-linear multiphoton-induced ablation processes occur [38, 43-47]. The dimensions of the interaction volume are defined by the diffraction limit of the optical system. Since the transverse diameter of the focus is in the order of $\sim 1 \mu \mathrm{m}$, the technique is able to prune dendrites with similar diameter while keeping the rest of the neuron's dendritic tree and its surrounding extracellular matrix intact [26]. Maintaining a diffraction limited focus when cutting dendrites in deeper regions of the brain tissue can also be achieved by pre-compensating the optical aberrations caused by neuronal structures in the cortical tissue [48]. Thicker dendrites can also be pruned by transversely moving the focus across the dendrite during a longer exposure time. The technique has been used to perform axotomy, where axons of L5PNs were cut to study the generation of action potentials [49]. Note, however, that the cutting parameters, such as exposure time and laser power need to be regulated to facilitate an effective cut while avoiding damage of the surrounding tissue that can affect cell viability [26]. Here, we have successfully used the technique to prune and investigate the capacity of oblique dendrites to evoke D-spikes. After cutting, the cells regain their 
function and their ability to fire action potentials following current injection. While our experiments were done in acute brain slices (in vitro), recent work by Yamaguchi et al. [50] demonstrated the use of the technique for deep (up to $\sim 550 \mu \mathrm{m}$ in depth from the surface of the mouse brain) focal dissection of apical and basal dendrites of L5PNs in vivo. Hence, in combination with other optical tools $[39,51]$, the ultrafast laser scalpel can potentially be applied to study D-spikes in an intact rodent brain.

\section{CONCLUSION}

In conclusion, we propose a technique to analyze branch-specific D-spikes in cortical pyramidal neurons. D-spikes that occur only on specific branches are difficult to analyze especially in complex dendritic arborizations where the application of pharmacological agents or dendritic pinching technique could affect neighboring dendrites. We performed highly targeted dendrotomy via a non linear optical ablation process using a tightly focused femtosecondpulse laser. We confined the interaction within the targeted dendrite thereby keeping the rest of the dendritic tree intact and the neuron remains viable. Our in vitro experiments were guided by numerical simulations, which confirmed our hypothesis that oblique branches have varying influence on neuronal function. While our proposed technique is irreversible, the technique can provide insights as to the role of certain dendrites in branch-specific computing mechanisms of individual neurons. Such goal can be achieved by performing systematic dendrotomy of oblique dendrites from different types of pyramidal neurons and classifying their corresponding outputs. Moreover, the ability to shape dendritic morphology to influence the output could also be used to study the broader roles of pyramidal neurons in brain circuits involving neuronal populations. D-spikes are critical computing attributes in pyramidal neurons and identifying their roles can facilitate a bottom-up approach to understand how the brain processes information during sensory perception, learning and memory.

\section{DATA AVAILABILITY STATEMENT}

The raw data supporting the conclusions of this article will be made available by the authors, without undue reservation.

\section{REFERENCES}

1. London M, Häusser M. Dendritic computation. Annu Rev Neurosci (2005) 28: 503-32. doi:10.1146/annurev.neuro.28.061604.135703.

2. Major G., Larkum ME, Schiller J. Active properties of neocortical pyramidal neuron dendrites. Annu Rev Neurosci (2013) 36:1-24. doi:10.1146/annurev-neuro-062111-150343.

3. Stuart GJ, Spruston N. Dendritic integration: 60 years of progress. Nat Neurosci (2015) 18(12):1713-21. doi:10.1038/nn.4157.

4. Larkum ME, Zhu JJ, Sakmann B. A new cellular mechanism for coupling inputs arriving at different cortical layers. Nature (1999) 398(6725):338-41. doi:10. $1038 / 18686$.

5. Schaefer AT, Larkum ME, Sakmann B, Roth A. Coincidence detection in pyramidal neurons is tuned by their dendritic branching pattern. J Neurophysiol (2003) 89(6):3143-54. doi:10.1152/jn.00046.2003.

\section{ETHICS STATEMENT}

The handling of rats was performed according to the protocol approved by the Animal. Experimentation Ethics Committee of the Australian National University (Ethics protocol Number: A2018/35, Principal Investigator VRD; Understanding the role of apical obliques in neuronal function). Co-Investigator $\mathrm{MC}$ has undergone trainings for rat handling and surgery to prepare samples for the in vitro experiment. Sample preparation and data gathering were performed in a Physical Containment Level 2 (PC2) Laboratory.

\section{AUTHOR CONTRIBUTIONS}

VD conceptualized the problem. VD built the microscope with advice from $\mathrm{H}-\mathrm{AB}$. $\mathrm{MC}$ performed the simulation and experiments. $\mathrm{MC}$ lead the writing of the manuscript with help from $\mathrm{H}-\mathrm{AB}$ and $\mathrm{VD}$.

\section{FUNDING}

This work was primarily funded by the National Health and Medical Research Council (PG1105944) and supported by the Australian Research Council (ARC) Discovery Project (DP140101555) and the ARC Centre of Excellence for Integrative Brain Function (CIBF).

\section{ACKNOWLEDGMENTS}

We thank Greg Stuart for the comments and guidance he provided. We also thank John Bekkers for the valuable discussions on dendrotomy.

\section{SUPPLEMENTARY MATERIAL}

The Supplementary Material for this article can be found online at: https://www.frontiersin.org/articles/10.3389/fphy.2020.600971/ full\#supplementary-material

6. Spruston N. Pyramidal neurons: dendritic structure and synaptic integration. Nat Rev Neurosci (2008) 9(3):206-21. doi:10.1038/nrn2286.

7. Sakmann B. From single cells and single columns to cortical networks: dendritic excitability, coincidence detection and synaptic transmission in brain slices and brains. Exp Physiol (2017) 102(5):489-521. doi:10.1113/ep085776.

8. Amitai Y, Friedman A, Connors BW, Gutnick MJ. Regenerative activity in apical dendrites of pyramidal cells in neocortex. Cerebr Cortex (1993) 3(1): 26-38. doi:10.1093/cercor/3.1.26.

9. Kim HG, Connors B. Apical dendrites of the neocortex: correlation between sodium- and calcium-dependent spiking and pyramidal cell morphology. J Neurosci (1993) 13(12):5301-11. doi:10.1523/jneurosci.13-12-05301. 1993.

10. Schiller J, Schiller Y, Stuart G, Sakmann B. Calcium action potentials restricted to distal apical dendrites of rat neocortical pyramidal neurons. J Physiol (1997) 505(Pt 3):605-16. doi:10.1111/j.1469-7793.1997.605ba.x. 
11. Kamondi A, Acsády L, Buzsáki G. Dendritic spikes are enhanced by cooperative network activity in the intact hippocampus. J Neurosci (1998) 18(10):3919-28. doi:10.1523/jneurosci.18-10-03919.1998.

12. Golding NL, Spruston N. Dendritic sodium spikes are variable triggers of axonal action potentials in hippocampal CA1 pyramidal neurons. Neuron (1998) 21(5):1189-200. doi:10.1016/s0896-6273(00)80635-2.

13. Gasparini S, Migliore M, Magee JC. On the initiation and propagation of dendritic spikes in CA1 pyramidal neurons. J Neurosci (2004) 24(49): 11046-56. doi:10.1523/jneurosci.2520-04.2004.

14. Losonczy A, Magee JC. Integrative properties of radial oblique dendrites in hippocampal CA1 pyramidal neurons. Neuron (2006) 50(2):291-307. doi:10. 1016/j.neuron.2006.03.016.

15. Larkum ME, Kaiser KMM, Sakmann B. Calcium electrogenesis in distal apical dendrites of layer 5 pyramidal cells at a critical frequency of back-propagating action potentials. Proc Nat Acad Sci (1999) 96(25):14600-4. doi:10.1073/pnas.96.25.14600.

16. Larkum ME, Waters J, Sakmann B, Helmchen F. Dendritic spikes in apical dendrites of neocortical layer 2/3 pyramidal neurons. J Neurosci (2007) 27(34): 8999-9008. doi:10.1523/jneurosci.1717-07.2007.

17. Larkum ME, Nevian T, Sandler M, Polsky A, Schiller J. Synaptic integration in tuft dendrites of layer 5 pyramidal neurons: a new unifying principle. Science. (2009) 325(5941):756-60. doi:10.1126/science.1171958.

18. Schiller J, Major G, Koester HJ, Schiller Y. NMDA spikes in basal dendrites of cortical pyramidal neurons. Nature (2000) 404(6775):285-9. doi:10.1038/ 35005094.

19. Branco T, Clark BA, Häusser M. Dendritic discrimination of temporal input sequences in cortical neurons. Science (2010) 329(5999):1671-5. doi:10.1126/ science. 1189664.

20. Castañares ML, Ma H, Daria VR. "Non-linear Calcium events along apical obliques of layer 5 cortical pyramidal neurons." In Program No. 461.07/D7. Neuroscience meeting planner, San Diego, CA: Society for Neuroscience (2018)

21. Castañares MLG, Stuart G, Daria VR. Dendritic spikes in apical oblique dendrites of cortical layer 5 pyramidal neurons. bioRxiv. (2020) 18:2020. doi:10.1101/2020.08.15.252080

22. Frick A, Magee J, Koester HJ, Migliore M, Johnston D. Normalization of $\mathrm{Ca} 2+$ Signals by small oblique dendrites of CA1 pyramidal neurons. J Neurosci (2003) 23(8):3243-50. doi:10.1523/jneurosci.23-08-03243.2003.

23. Palmer LM, Shai AS, Reeve JE, Anderson HL, Paulsen O, Larkum ME. NMDA spikes enhance action potential generation during sensory input. Nat Neurosci (2014) 17(3):383-90. doi:10.1038/nn.3646.

24. Bekkers JM, Häusser M. Targeted dendrotomy reveals active and passive contributions of the dendritic tree to synaptic integration and neuronal output. Proc Natl Acad Sci (2007) 104(27):11447-52. doi:10.1073/pnas. 0701586104.

25. Metz AE, Jarsky T, Martina M, Spruston N. R-type calcium channels contribute to afterdepolarization and bursting in hippocampal CA1 pyramidal neurons. J Neurosci (2005) 25(24):5763-73. doi:10.1523/jneurosci.0624-05.2005.

26. Go MA, Choy JM, Colibaba AS, Redman S, Bachor HA, Stricker C, et al.. Targeted pruning of a neuron's dendritic tree via femtosecond laser dendrotomy. Sci Rep (2016) 6:19078. doi:10.1038/srep19078.

27. Hines ML, Davison AP, Muller E. NEURON and Python. Front Neuroinform (2009) 3:1. doi:10.3389/neuro.11.001.2009.

28. Shai AS, Anastassiou CA, Larkum ME, Koch C. Physiology of layer 5 pyramidal neurons in mouse primary visual cortex: coincidence detection through bursting. PLoS Comput Biol (2015) 11(3):24. doi:10.1371/journal.pcbi. 1004090.

29. Morse TM, Carnevale NT, Mutalik PG, Migliore M, Shepherd GM. Abnormal excitability of oblique dendrites implicated in early Alzheimer's: a computational study. Front Neural Circ (2010) 4:601-17.

30. Migliore M, Ferrante M, Ascoli GA. Signal propagation in oblique dendrites of CA1 pyramidal cells. J Neurophysiol (2005) 94(6):4145-55. doi:10.1152/jn. 00521.2005

31. Zhou WL, Short SM, Rich MT, Oikonomou KD, Singh MB, Sterjanaj EV, Antic SD. Branch specific and spike-order specific action potential invasion in basal, oblique, and apical dendrites of cortical pyramidal neurons. Neurophotonics (2015) 2(2):021006. doi:10.1117/1.NPh.2.2. 021006.

32. Harnett MT, Xu N-L, Magee JC, Williams SR. Potassium channels control the interaction between active dendritic integration compartments in layer 5 cortical pyramidal neurons. Neuron (2013) 79(3):516-29. doi:10.1016/j. neuron.2013.06.005.

33. Castanares ML, Stuart GJ, Daria VR. Holographic functional calcium imaging of neuronal circuit activity. In: F-J Kao, G Keiser, A Gogoi, editors Advanced optical methods for brain imaging. Singapore: Springer (2019)

34. Go MA, To MS, Stricker C, Redman S, Bachor HA, Stuart GJ, et al. Fourdimensional multi-site photolysis of caged neurotransmitters. Front Cell Neurosci (2013) 7:231. doi:10.3389/fncel.2013.00231.

35. Mainen ZF, Sejnowski TJ. Influence of dendritic structure on firing pattern in model neocortical neurons. Nature (1996) 382(6589):363-6. doi:10.1038/ 382363a0.

36. Vetter P, Roth A, Häusser M. Propagation of action potentials in dendrites depends on dendritic morphology. J Neurophysiol (2001) 85(2):926-937. doi:10.1152/jn.2001.85.2.926.

37. Kirson ED, Yaari Y. A novel technique for micro-dissection of neuronal processes. J Neurosci Methods (2000) 98(2):119-22. doi:10.1016/s01650270(00)00194-1.

38. Sacconi L, O'Connor RP, Jasaitis A, Masi A, Buffelli M, Pavone FS. In vivo multiphoton nanosurgery on cortical neurons. J Biomed Opt. (2007) 12(5): 050502. doi:10.1117/1.2798723.

39. Go MA, Daria VR. Light-neuron interactions: key to understanding the brain. J Optic (2017) 19(2):023002. doi:10.1088/2040-8986/19/2/023002.

40. Kampa BM, Stuart GJ. Calcium spikes in basal dendrites of layer 5 pyramidal neurons during action potential bursts. J Neurosci (2006) 26(28):7424-32. doi:10.1523/jneurosci.3062-05.2006.

41. Nevian T, Larkum ME, Polsky A, Schiller J. Properties of basal dendrites of layer 5 pyramidal neurons: a direct patch-clamp recording study. Nat Neurosci (2007) 10(2):206-14. doi:10.1038/nn1826.

42. Losonczy A, Makara JK, Magee JC. Compartmentalized dendritic plasticity and input feature storage in neurons. Nature (2008) 452(7186):436-441. doi: 10.1038 /nature06725.

43. König K, Riemann I, Fritzsche W. Nanodissection of human chromosomes with near-infrared femtosecond laser pulses. Opt Lett. (2001) 26(11):819-21. doi:10.1364/ol.26.000819.

44. Yanik MF, Cinar H, Cinar HN, Chisholm AD, Jin Y, Ben-Yakar A. Functional regeneration after laser axotomy. Nature (2004) 432(7019):822. doi:10.1038/ 432822a.

45. Ando J, Bautista G, Smith N, Fujita K, Daria VR. Optical trapping and surgery of living yeast cells using a single laser. Rev Sci Instrum (2008) 79(10):103705. doi:10.1063/1.2999542.

46. Bourgeois F, Ben-Yakar A. Femtosecond laser nanoaxotomy properties and their effect on axonal recovery in C. elegans. Opt Express (2008) 16(8):5963. doi:10.1364/oe.16.005963.

47. Allegra Mascaro AL, Sacconi L, Pavone FS. Multi-photon nanosurgery in live brain. Front Neuroenerg (2010) 2:28. doi:10.3389/fnene.2010.00021.

48. Choy JM, Sané SS, Lee WM, Stricker C, Bachor HA, Daria VR. Improving focal photostimulation of cortical neurons with pre-derived wavefront correction. Front Cell Neurosci (2017) 11:105. doi:10.3389/fncel.2017.00105.

49. Kole MHP. First node of Ranvier facilitates high-frequency burst encoding. Neuron. (2011) 71(4):671-82. doi:10.1016/j.neuron.2011.06.024.

50. Yamaguchi K, Kitamura R, Kawakami R, Otomo K, Nemoto T. In vivo twophoton microscopic observation and ablation in deeper brain regions realized by modifications of excitation beam diameter and immersion liquid. PLoS One. (2020) 15(8):e0237230. doi:10.1371/journal.pone.0237230.

51. Daria VR, Bachor H-A. Using light to probe neuronal function. Epl (2015) 111(3):38001. doi:10.1209/0295-5075/111/38001. 7

Conflict of Interest: The authors declare that the research was conducted in the absence of any commercial or financial relationships that could be construed as a potential conflict of interest.

Copyright $\odot 2020$ Castañares, Bachor and Daria. This is an open-access article distributed under the terms of the Creative Commons Attribution License (CC BY). The use, distribution or reproduction in other forums is permitted, provided the original author(s) and the copyright owner(s) are credited and that the original publication in this journal is cited, in accordance with accepted academic practice. No use, distribution or reproduction is permitted which does not comply with these terms. 